\title{
DISTRIBUIÇÃO DE MERCÚRIO TOTAL COMO INDICADOR DE POLUIÇÃO URBANA E INDUSTRIAL NA COSTA BRASILEIRA
}

Rozane V. Marins*, Francisco José de Paula Filho e Saulo Robério Rodrigues Maia

Instituto de Ciências do Mar, Universidade Federal do Ceará, Av. Abolição 3207, 60165-081 Fortaleza - CE

Luiz Drude de Lacerda

Departamento de Geoquímica, Universidade Federal Fluminense, Outeiro de São João Batista s/n, 24020-007 Niterói - RJ

Wanessa Sousa Marques

Centro de Ciências, Universidade Federal do Ceará, Campus do Pici, 60455-970 Fortaleza - CE

Recebido em 9/6/03; aceito em 9/10/03; publicado na web em 17/6/04

\begin{abstract}
TOTAL MERCURY DISTRIBUTION AS A PROXY OF URBAN AND INDUSTRIAL POLLUTION ALONG THE BRAZILIAN COAST. The monitoring of the pollution status of the Brazilian coast is hampered by the lack of a consistent environmental indicator. We propose $\mathrm{Hg}$ as an environmental proxy to anthropogenic pollution of coastal regions due to its absence in the Brazilian geology, its ubiquity in anthropogenic effluents, and its toxicity. Available data suggest $\mathrm{Hg}$ "backgrounds" in coastal sediments of 1-10 $\mathrm{ng} \mathrm{g}^{-1}$, in the East and 15-30 $\mathrm{ng} \mathrm{g}^{-1}$ in the South. We suggest short sediment cores to establish these values estimating a geo-accumulation index to compare all areas. The distribution of index values showed the majority of southern coast at least moderately contaminated whereas many eastern areas are still pristine.
\end{abstract}

Keywords: environmental proxy; mercury; geo-accumulation index

\section{INTRODUÇÃo}

O crescente controle das principais fontes pontuais de poluição têm resultado no aumento relativo da importância das fontes difusas, tipicamente de difícil controle e monitoramento. Nas regiões costeiras de um modo geral, o impacto das fontes difusas de poluição se dá através da integração de efluentes em bacias de drenagem da região. Isto dificulta a caracterização de fontes e poluentes singulares e resulta na necessidade de se estabelecer indicadores consistentes e capazes de monitorarem alterações temporais na magnitude dessas fontes e sua variabilidade espacial ao longo de extensos trechos do litoral.

Em um programa internacional recente de avaliação das atividades antrópicas das bacias de drenagem que modificam as regiões costeiras $^{1,2}$ observou-se que a despeito do uso de índices que permitem avaliar e comparar o grau de contaminação de regiões costeiras de climas temperados, como o teor de oxigênio dissolvido em águas estuarinas e costeiras ${ }^{3}$, há falta do uso de indicadores da contaminação ambiental em clima tropical. Por exemplo, o teor de oxigênio dissolvido em sistemas aquáticos é muito utilizado em regiões de clima temperado para determinar a eutrofização cultural. Em clima tropical, entretanto, esse parâmetro pode apresentar uma grande variabilidade devida, por exemplo, a diferenças de temperaturas das águas que banham a costa brasileira, além do fato de que, devido à exuberância da cobertura vegetal de vários dos ecossistemas costeiros tropicais, há grande disponibilidade natural de matéria orgânica para o meio aquoso, aliada a elevadas taxas de insolação e temperaturas, proporcionando uma elevada taxa de degradação da matéria orgânica, o que naturalmente, ou seja, sem interferência de atividades antrópicas, pode levar a diminuições significativas do conteúdo de oxigênio dissolvido em águas costeiras tropicais. O fenômeno em águas fluviais tropicais é bem conhecido ${ }^{4}$.

\footnotetext{
*e-mail: rmarins@labomar.ufc.br
}

Um critério que gere comparabilidade de resultados e prognósticos da situação ambiental da costa brasileira fornecendo definições que apontem soluções deve, segundo norma do Governo Federal ${ }^{5}$ para a poluição marinha, considerar três tópicos: a) as principais fontes de poluição; b) os efeitos sobre as atividades econômicas e c) as estratégias e ações nacionais e internacionais para o decréscimo dos impactos sobre o ambiente marinho.

\section{SUMÁRIO DAS CARACETRÍSTICAS AMBIENTAIS DA COSTA BRASILEIRA}

A zona costeira brasileira compreende uma faixa terrestre de $20 \mathrm{~km}$ e uma faixa marítima de 6 milhas $(11,1 \mathrm{~km})$ de extensão a partir da linha de costa. Essa região vem sendo ocupada desde os tempos coloniais e concentra parcela significativa da população. Em alguns estados litorâneos essa densidade demográfica pode se ampliar como no caso do Ceará, onde 48,9\% da população vivem na região litorânea ${ }^{6}$.

A classificação da região costeira brasileira vem sendo feita segundo diferentes parâmetros de cunho geológico, climático, geográfico, oceanográfico, sedimentológico. Ou seja, existem padrões que se repetem e que permitem a classificação da costa brasileira em porções, parcelas que apresentam similaridades classificatórias, entretanto essas avaliações não descrevem uma comparabilidade sobre a qualidade ambiental das diferentes regiões.

Recentemente uma tipologia para a costa brasileira foi propos$\mathrm{ta}^{7}$, adotando o conceito de LME ("Large Marine Ecosystem") que define regiões extensas de acordo com suas similaridades batimétricas, hidrográficas, de produtividade e de dependência trófica das cadeias alimentares costeiras. De acordo com essa proposição, a costa brasileira é subdividida nas porções: Norte, Leste e Sul. As porções Norte e Sul são controladas particularmente pela topografia da costa e pelas fontes continentais de materiais, sustentando uma menor diversidade da cadeia alimentar e a mais alta produtividade. A porção Leste da LME brasileira é caracterizada como um ambiente tipicamente 
oligotrófico, dominado pelos limites das correntes oceânicas, com elevada biodiversidade e baixa produtividade.

A porção Leste da LME brasileira corresponde geograficamente à região costeira que se estende do estuário do Rio Parnaíba ao Cabo de São Tomé. As águas superficiais apresentam temperaturas médias de $28^{\circ} \mathrm{C}$ e salinidade de $36,8^{8}$. É uma região de mesomarés e de águas dominadas pela corrente Equatorial Sul (SEC). Os rios da região são fortemente influenciados pela sazonalidade do clima, embora na região costeira os índices pluviométricos possam ser elevados. Na região de Fortaleza ${ }^{9}$, por exemplo, o índice pluviométrico médio é de $1550 \mathrm{~mm}$. Comparada às regiões Norte e Sul essa região é a mais oligotrófica das águas costeiras do Brasil. O litoral é dominado pela Formação Barreiras, terciária, que aflora em porções extensas ao longo da costa. Dunas, mangues e bancos de corais apontam ao longo de toda a porção leste da LME brasileira? .

A porção Sul da LME brasileira está incluída na região denominada de USWA ("Upper Southwest Atlantic Ocean") ${ }^{10}$, uma região influenciada por duas correntes oceânicas. A corrente Brasil que flui do Norte para o Sul e a corrente das Malvinas que flui em direção ao Norte. Ambas as correntes estão restritas a profundidades menores que $1500 \mathrm{~m}$ e se encontram na região próxima à foz do Rio Prata, criando uma forte e larga zona frontal, conhecida como de Convergência Atlântica Subtropical, que separa as águas subtropicais e subantárticas. Nessa região a pesca comercial é uma das mais importantes atividades comerciais. A captura é de cerca de $2.136 .000 \mathrm{t}$ além da captura de 362.500 t de crustáceos, sendo que o Brasil é responsável por cerca de $33 \%$ da captura total, principalmente de sardinhas e crustáceos ${ }^{11}$. Nessa região há o domínio do clima tropical e do clima temperado nas latitudes mais ao Sul, ocorrendo dois grandes grupos de ecossistemas costeiros de grande importância ecológica: os mangues nas áreas tropicais e os banhados nas regiões de clima temperado. A área tropical inclui os litorais do Rio de Janeiro e São Paulo, que recebem águas das bacias de drenagens mais industrializadas do País. A região temperada inclui o litoral do Paraná, Santa Catarina e do Rio Grande do $\mathrm{Sul}^{10}$.

Sobre a porção Norte, quaternária, da LME brasileira não há informações disponíveis sobre o teor de $\mathrm{Hg}$ em sedimentos estuarinos e costeiros, parâmetro utilizado nesse trabalho. Dessa forma, essa parte do litoral não será avaliada. A despeito disso são bem relatados os níveis de contaminação por mercúrio no ambiente fluvial amazônico.

\section{METAIS PESADOS COMO INDICADORES AMBIENTAIS - O CASO DO MERCÚRIO}

Desde a década de 70 a extensão da poluição global vem sendo testada através de medidas de metais pesados, comparando-se inicialmente o consumo desses metais com a concentração natural dos elementos em sedimentos não poluídos, denominando-se esse valor de Índice Relativo de Poluição Potencial ${ }^{12}$. Posteriormente, foi aplicado o Índice de Geoacumulação $0^{13}$, que conferiu um caráter maior de comparabilidade aos estudos de poluição dos sistemas aquáticos por metais pesados. Mais recentemente, a medida da poluição em sistemas aquáticos foi estimada através da determinação da média da carga de metal disponibilizada para os sedimentos desses sistemas, e foram considerados os níveis pré-existentes desses metais na era préindustrial, obtidos pela comparação dos teores desses metais ao longo de perfis de testemunhos sedimentares. Esta medida foi designada de Fator de Enriquecimento do Metal entre a era pré-industrial e a atual ${ }^{14}$. No caso da costa brasileira há relatos de contaminação por metais pesados em várias regiões, como resultado das elevadas taxas de emissão por atividades industriais e urbanas concentradas nas bacias de drenagens e na região costeira desde a década de 80. Entretanto, são raros os estudos comparativos.
Desde o início das avaliações da poluição por metais, o mercúrio vem sendo apontado como um dos metais de importância para o monitoramento global da poluição antrópica ${ }^{14}$, devido a várias características, tais como: a) sua extensa utilização - na década de 70 eram conhecidos mais de seiscentos usos de $\mathrm{Hg}$ nas indústrias ${ }^{15}$; b) toxidez - o mercúrio em sua forma de metilada possui seletividade para atacar o sistema nervoso central e suas estruturas mais evoluídas, tal como o córtex cerebral ${ }^{16}$ e c) comportamento no meio ambiente - avaliações em sistema costeiro subtropical demonstraram que, diferentemente de outros metais, pequena parcela de mercúrio emitida para o ambiente é retida nos sedimentos, a maior parte encontra no meio aquoso mecanismos que facilitam sua permanência na coluna d'água ${ }^{17-20}$.

No caso da costa brasileira, o Fator de Enriquecimento, medido através da comparação entre os teores de mercúrio em sedimentos costeiros da era pré-industrial e atual, pode fornecer uma indicação importante e sensível da presença e magnitude de atividades urbanas e industriais e de seus efeitos deletérios ao ambiente costeiro. Esta informação, acrescida das caracterizações oceanográficas existentes, incluindo dados sobre o potencial de produção biológica, poderá fornecer um parâmetro de qualidade ambiental com caráter espacial e temporal.

\section{FONTES DE MERCÚRIO PARA O AMBIENTE COSTEIRO}

Processos naturais como a ressuspensão de partículas do solo pelos ventos, salssugem, emanações vulcânicas, queimadas de florestas e emanações do solo e águas superficiais podem contribuir para as emissões naturais de mercúrio. O intemperismo também pode disponibilizar o metal contido em rochas, entretanto no Brasil não são conhecidas regiões de mineralização de mercúrio, com exceção de uma pequena área próxima a Ouro Preto ${ }^{21}$.

As principais emissões antropogênicas de mercúrio incluem aquelas concentradas localmente devido a despejos, tais como descargas de efluentes ou resíduos que ocorriam em antigas plantas eletroquímicas para a produção de cloro-soda, e descargas difusas tais como aquelas associadas à produção de energia, como na combustão de carvão e derivados de petróleo ${ }^{22}$, ou queima de lixo, principalmente do lixo hospitalar ${ }^{23}$. Recentemente a contaminação por mercúrio do material particulado de esgotos industriais, domésticos e do esgotamento central da cidade do Rio Grande foi avaliada ${ }^{24}$. A faixa de concentração de mercúrio no material particulado desses efluentes variou de 0,12 a $21,14 \mathrm{mg} \mathrm{kg}^{-1}$. A maior concentração de mercúrio no material particulado avaliado foi encontrada em efluente do esgotamento central da cidade de Rio Grande e o valor mais baixo, em efluente industrial de uma fábrica de fertilizantes. $\mathrm{O}$ segundo grupo em ordem decrescente das concentrações de mercúrio no material particulado foi o de medidas realizadas em material de drenagens a céu aberto. Segundo os autores, esses valores podem representar a contaminação das drenagens pelo chorume dos lixões despejados em suas margens.

A atmosfera desempenha, no caso da contaminação por mercúrio, um importante papel de corpo receptor tanto das emissões naturais como antrópicas. Devido a suas propriedades físico-químicas e meteorológicas a atmosfera pode distribuir e redistribuir as emissões recebidas, atuando como uma importante fonte difusa de mercúrio para solos e drenagens ${ }^{25-27}$. Ou seja, embora os processos industriais tenham substituído o mercúrio como matéria-prima de vários processos produtivos, o metal permanece sendo emitido principalmente por fontes difusas e como constituinte traço de efluentes urbanos e domésticos. 


\section{CONCENTRAÇÕES DE MERCÚRIO EM SEDIMENTOS SUPERFICIAIS NA COSTA BRASILEIRA}

Vários autores desde a década de 80 têm reportado contaminações de sedimentos superficiais em várias regiões da costa brasileira. As Tabelas 1a e 1b mostram esses resultados.

Esses resultados são em grande parte comparáveis, uma vez que a maioria refere-se à fração sedimentar $<63 \mu \mathrm{m}$, considerada a mais adequada nas avaliações de contaminantes, tendo em vista que: os metais preferencialmente se associam ao material fino que contem argilas, silte e matéria orgânica particulada, que são os principais carreadores de metais nos ecossistemas aquáticos; essa fração é similar ao material carreado em suspensão, que representa o material de maior importância para o transporte a longas distâncias no meio aquático; o peneiramento nessa faixa não altera a concentração de poluentes, pois os metais preservam-se durante o processo de separação granulométrica, mesmo quando feito a úmido e com água do local de coleta.

Reforçando as hipótese sobre o uso da fração $<63 \mu \mathrm{m}$, grande número de avaliações ambientais de metais pesados em sedimentos têm sido efetivadas nessa fração, desde a década de 80 , em todo o mundo $^{36}$. Esses dados apontam valores mínimos detectáveis no litoral Leste da LME brasileira na ordem de 1 a $10 \mathrm{ng} \mathrm{g}^{-1}$ para a fração $<63 \mu \mathrm{m}$, enquanto que na fração $<2 \mu \mathrm{m}$ o valor mínimo reportado é de $60 \mathrm{ng} \mathrm{g}^{-1}$. Essa variabilidade de teores dentro de diferentes faixas mineralógicas é esperada porque cada vez que se concentra mais o material fino há tendência de se concentrar mais o teor de metais pesados $^{14,37}$.

Os valores de $\mathrm{Hg}$ encontrados na fração $<2 \mu \mathrm{m}$ no litoral Leste, entretanto, não representam contaminação dos ambientes estudados pois foi verificada uma depleção dos valores de mercúrio quando comparados aos teores do folhelho médio $\left(40 \mathrm{ng} \mathrm{g}^{-1}\right)^{30}$. Porém, observando-se os demais valores encontrados na fração $<63 \mu \mathrm{m}$ verifica-se que o valor médio mundial do folhelho deve estar superestimando os valores de "background" da região Leste da LME brasileira, pois se encontram valores na ordem de unidades de $\mathrm{ng} \mathrm{g}^{-1} \mathrm{em}$ sedimentos superficiais da região.

Por outro lado, observa-se no litoral Sul da LME brasileira que os valores mínimos encontrados são maiores. Mesmo tendo sido analisadas a fração total dos sedimentos, a faixa mínima reportada está compreendida entre 20 a $30 \mathrm{ng} \mathrm{g}^{-1}$. Um único valor de $10 \mathrm{ng} \mathrm{g}^{-1}$ foi encontrado em sedimentos de mangue. Entretanto, tem sido observado que os sedimentos do mangue apresentam valores menores do que os reportados em canais de maré e planícies lamosas em frente a esses mesmos manguezais, ou seja, parece haver uma retenção prévia de metais antes que alcancem os sedimentos do mangue ${ }^{35,38-40}$. Além disso é conhecido que a retenção do metal no manguezal e até
Tabela 1a. Resultados de mercúrio em sedimentos superficiais reportados na literatura para a porção Leste da LME brasileira

\begin{tabular}{|c|c|c|}
\hline \multicolumn{3}{|c|}{$\begin{array}{l}\text { Litoral Leste da LME brasileira, segundo a tipologia } \\
\text { de Ekau e Knoppers }{ }^{7}\end{array}$} \\
\hline Local amostrado & $\begin{array}{l}\text { Teor de } \mathrm{Hg} \\
\left(\mathrm{ng} \mathrm{g}^{-1}\right)\end{array}$ & $\begin{array}{l}\text { Fração analisada } \\
\text { do sedimento }\end{array}$ \\
\hline Baía de Todos os Santos $(\mathrm{BA})^{28}$ & 10 a 820 & total \\
\hline Foz do Rio Botafogo $(\mathrm{PE})^{29}$ & 2.300 a 3.100 & total \\
\hline Foz do Rio Carrapicho $(\mathrm{PE})^{29}$ & 600 a 1.300 & total \\
\hline Foz do R. Parnaíba $(\mathrm{PI})^{30}$ & 60 & $<2 \mu \mathrm{m}$ \\
\hline Foz do R. Timonha $(\mathrm{CE})^{30}$ & 150 & $<2 \mu \mathrm{m}$ \\
\hline Foz do R. Coreau $(\mathrm{CE})^{30}$ & 150 & $<2 \mu \mathrm{m}$ \\
\hline Foz do R. Acarau $(\mathrm{CE})^{30}$ & 130 & $<2 \mu \mathrm{m}$ \\
\hline Foz do Curu $(\mathrm{CE})^{30}$ & 120 & $<2 \mu \mathrm{m}$ \\
\hline Foz do R. Pacoti $(\mathrm{CE})^{30}$ & 100 & $<2 \mu \mathrm{m}$ \\
\hline Foz do R. Choro $(\mathrm{CE})^{30}$ & 100 & $<2 \mu \mathrm{m}$ \\
\hline Foz do R. Pirangi $(\mathrm{CE})^{30}$ & 100 & $<2 \mu \mathrm{m}$ \\
\hline Foz do R. Jaguaribe $(\mathrm{CE})^{30}$ & 80 & $<2 \mu \mathrm{m}$ \\
\hline Foz do R. Açu $(\mathrm{RGN})^{30}$ & 100 & $<2 \mu \mathrm{m}$ \\
\hline Foz do R. Paraíba (PB) ${ }^{30}$ & 200 & $<2 \mu \mathrm{m}$ \\
\hline Foz do R. São Francisco $(\mathrm{SE})^{30}$ & 130 & $<2 \mu \mathrm{m}$ \\
\hline Foz do R. Sergipe $(\mathrm{SE})^{30}$ & 250 & $<2 \mu \mathrm{m}$ \\
\hline Foz da Vasa Barris ${ }^{30}$ & 100 & $<2 \mu \mathrm{m}$ \\
\hline Foz do R. Piauíio & 150 & $<2 \mu \mathrm{m}$ \\
\hline Foz do R. Real ${ }^{30}$ & 130 & $<2 \mu \mathrm{m}$ \\
\hline Foz do R. Itapicuru ${ }^{30}$ & 100 & $<2 \mu \mathrm{m}$ \\
\hline Foz do R. Jiquirica ${ }^{30}$ & 300 & $<2 \mu \mathrm{m}$ \\
\hline Foz do R. Negro (BA) ${ }^{30}$ & 250 & $<2 \mu \mathrm{m}$ \\
\hline Foz do R. das Contas $(\mathrm{BA})^{30}$ & 180 & $<2 \mu \mathrm{m}$ \\
\hline Foz do R. Pardo $(\mathrm{BA})^{30}$ & 200 & $<2 \mu \mathrm{m}$ \\
\hline Foz do R. Jequitinhonha (BA) ${ }^{30}$ & 280 & $<2 \mu \mathrm{m}$ \\
\hline Foz do R. Jacuru Norte $(\mathrm{BA})^{30}$ & 200 & $<2 \mu \mathrm{m}$ \\
\hline Foz do R. Itanhem (BA) $)^{30}$ & 300 & $<2 \mu \mathrm{m}$ \\
\hline Foz do R. Mucuri (BA) ${ }^{30}$ & 200 & $<2 \mu \mathrm{m}$ \\
\hline Foz do R. São Mateus (ES) ${ }^{30}$ & 200 & $<2 \mu \mathrm{m}$ \\
\hline Foz do R. Doce $(\mathrm{ES})^{30}$ & 160 & $<2 \mu \mathrm{m}$ \\
\hline R. Ceará-Maranguapinho $(\mathrm{CE})^{31}$ & 2 a 40 & $<63 \mu \mathrm{m}$ \\
\hline R. Ceará-Maranguapinho $(\mathrm{CE})^{32}$ & 1 a 45 & $<63 \mu \mathrm{m}$ \\
\hline R. Ceará $(\mathrm{CE})^{33}$ & 21 a 40 & $<63 \mu \mathrm{m}$ \\
\hline R. Cocó $(\mathrm{CE})^{33}$ & 48 & $<63 \mu \mathrm{m}$ \\
\hline R. Pacoti $(\mathrm{CE})^{33}$ & 5 & $<63 \mu \mathrm{m}$ \\
\hline R. Jaguaribe $(\mathrm{CE})^{33}$ & 10 & $<63 \mu \mathrm{m}$ \\
\hline
\end{tabular}

Tabela 1b. Resultados de mercúrio em sedimentos superficiais reportados na literatura para a porção Sul da LME brasileira Litoral Sul da LME brasileira, segundo a tipologia de Ekau e Knoppers ${ }^{7}$

\begin{tabular}{|c|c|c|}
\hline Local amostrado & Teor de $\mathrm{Hg}\left(\mathrm{ng} \mathrm{g}^{-1}\right)$ & Fração analisada do sedimento \\
\hline Rios da Baía de Sepetiba $(\mathrm{RJ})^{17}$ & 36 a 197 & Total \\
\hline Baía de Sepetiba $(\mathrm{RJ})^{17}$ & 17 a 163 & Total \\
\hline R. São João de Meriti (RJ) ${ }^{34}$ & 32 a 3.380 & Total \\
\hline Sedimentos de mangue dos Rios Cubatão e Mogi (SP) ${ }^{35}$ & 10 a 30 & Total \\
\hline Sedimentos da planície de mangue dos Rios Cubatão e Mogi (SP) ${ }^{35}$ & 20 a 60 & Total \\
\hline Lagoa dos Patos $(\mathrm{RS})^{24}$ & 20 a 1.784 & Total \\
\hline Estuário da Baía de Santos (SP) ${ }^{10}$ & 575 & - \\
\hline Estuário da Baía de São Vicente $(\mathrm{SP})^{10}$ & 285 & - \\
\hline Baía de Santos $(\mathrm{SP})^{10}$ & 67 & - \\
\hline
\end{tabular}


mesmo em planícies lamosas pode sofrer alterações ligadas à própria dinâmica do ecossistema e à atividade biológica existente ${ }^{41,42}$.

\section{VARIABILIDADE SAZONAL DOS TEORES TOTAIS DE MERCÚRIO EM SEDIMENTOS COSTEIROS NO SETOR LESTE DA LME BRASILEIRA}

As possíveis alterações dos teores de mercúrio total em sedimentos coletados nas duas diferentes estações climáticas que dominam o setor Leste da LME brasileira ${ }^{7}$ foram avaliadas, tomando-se como exemplo coletas feitas no estuário do Rio Jaguaribe e na costa da Região Metropolitana de Fortaleza (RMF), à época de chuvas e seca.

No estuário do Rio Jaguaribe os teores de mercúrio em sedimentos na fração fina $(<63 \mu \mathrm{m})$ variaram de 5,5 a 7,1 $\mathrm{ng} \mathrm{g}^{-1}$ no período de chuvas. Ao longo do estuário há uma pequena variação dos teores, provavelmente associada a uma variabilidade de carreadores geoquímicos e a uma diluição de sedimentos fluviais por sedimentos marinhos. Esse comportamento tem sido observado mesmo em áreas onde a contaminação por atividades antrópicas é significativa e pode se estender além da linha de costa por toda a extensão estuarina $^{34,35,43}$.

No período de estiagem, coletas feitas nos mesmos locais do estuário do Rio Jaguaribe mostraram um aumento dos teores de mercúrio em sedimentos, os valores variaram de 9,1 a 11,4 $\mathrm{ng} \mathrm{g}^{-1}$, demonstrando maior retenção do metal nesse período.

Comportamento similar foi observado na plataforma da RMF (CE), para a maioria dos pontos de coleta analisados.

No período de chuvas, os teores de $\mathrm{Hg}$ em sedimentos na maioria dos pontos de coleta na RMF (CE) variaram de 0,5 a $4,5 \mathrm{ng} \mathrm{g}^{-1}$ (Figura 1). Teores mais altos fora dessa faixa caracterizam a influência do Porto do Mucuripe (Estação 27), a contaminação da plataforma pela afluência do rio Ceará-Maranguapinho (Estação 11), previamente avaliado ${ }^{31,32}$, e a presença de fonte pontual de mercúrio através do efluente do emissário submarino de Fortaleza (Estação 2), corroborando resultados encontrados na avaliação de esgotamentos urbanos e domésticos na região Sul da LME brasileira ${ }^{24}$. Na época de seca, os teores variaram de 0,3 a 9,7 $\mathrm{ng} \mathrm{g}^{-1}$, sendo maiores, portanto, do que no período de chuvas. Na Estação localizada em frente a saída do emissário submarino de Fortaleza (Estação 2) e na Estação 27, no Porto do Mucuripe entretanto, houve decréscimo dos teores de $\mathrm{Hg}$ em sedimentos no período seco. $\mathrm{O}$ aumento na concentração de mercúrio nas duas estações deve estar refletindo a maior quantidade de mercúrio emitido por fontes pontuais no período de chuvas.

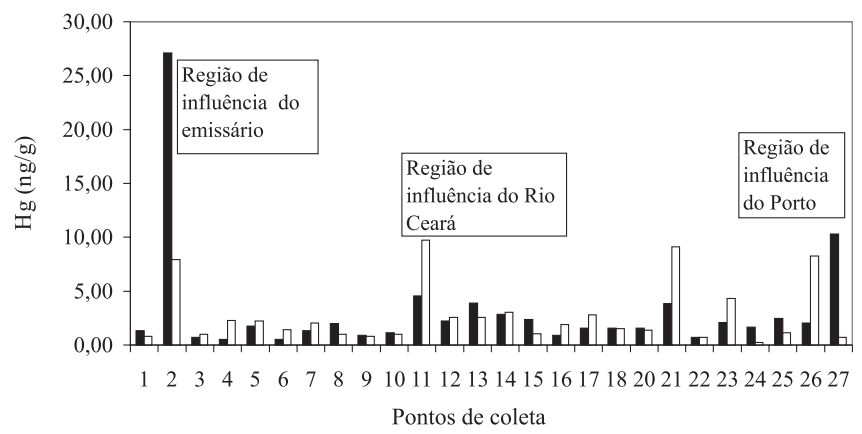

Figura 1. Distribuição espacial de Hg na Plataforma da Região Metropolitana de Fortaleza (RMF), Ceará, em época de chuvas $\mathbf{\square}$ e seca $\square$

Por outro lado, nas demais estações de coleta da RMF (CE) há uma variabilidade da distribuição dos teores máximos entre a estação de chuvas e seca. Essa variabilidade pode ser atribuída a fatores como o posicionamento dos pontos por GPS, que pode permitir que entre campanhas haja um erro da localização do ponto de até $40 \mathrm{~m}$ e a fatores climáticos que alteram as correntes marinhas na região entre as diferentes estações do ano e, conseqüentemente, o processo deposicional dos sedimentos costeiros.

\section{“BACKGROUNDS" REGIONAIS ATRAVÉS DE AVALIAÇÕES DE PERFIS SEDIMENTARES}

Para avaliar "backgrounds" regionais, isto é, valores básicos ou naturais, é necessário uma boa compilação de dados e um controle crítico que gerencie os resultados de forma abrangente, considerando as variabilidades do elemento ou substância em questão numa determinada matriz, sendo ilusória a idéia de "background" geoquímico global ${ }^{44}$ que teve importância nas primeiras avaliações geoquímicas, quando poucas medidas reais tinham sido feitas em diferentes partes do mundo.

Vários estudos têm reportado que a média mundial ("Clarke" geoquímico) e as concentrações médias de metais no folhelho são muito elevadas para representarem os níveis de "background" em diferentes bacias sedimentares ${ }^{45}$. Uma das soluções propostas desde o início das avaliações de metais pesados é definir limites de "background", através da avaliação dos teores existentes em sedimentos depositados nas bacias sedimentares em eras pré-industriais. Para determinar a profundidade em que esses sedimentos encontramse depositados na atualidade, é necessário conhecer as taxas de sedimentação das diferentes bacias.

Taxas de sedimentação maiores do que $2 \mathrm{~cm}$ ano $^{-1}$ foram medidas em situações extremas, em locais caracterizados pela canalização de drenagens e desmatamento intenso, que é uma das características de grandes áreas de urbanização. No Brasil, taxas de sedimentação variando de 1,3 a $2,2 \mathrm{~cm}$ ano $^{-1}$, relatadas para pontos específicos da Baía de Guanabara ${ }^{46}$ e Baía de Sepetiba ${ }^{47,48}$ no Estado do Rio de Janeiro, são consideradas elevadas $\left(>2 \mathrm{~cm} \mathrm{ano}^{-1}\right)$, e podem resultar em camadas muito espessas de sedimentos recentes, trazendo consigo a assinatura de fontes antrópicas recentes. Neste caso, testemunhos muito longos (> 1,0 m) são necessários para se atingir os valores pré-industriais. Tais testemunhos são de difícil obtenção e tornam problemática a determinação dos valores de "background". Entretanto, nas duas baías as taxas médias de sedimentação são até 10 vezes menores que os valores máximos.

Por outro lado, taxas de sedimentação muito baixas $\left(<0,01 \mathrm{~cm} \mathrm{ano}^{-1}\right)$ podem resultar na acumulação de $\mathrm{Hg}$ proveniente de longos períodos de sedimentação, incluindo períodos com variações significativas dos fluxos de $\mathrm{Hg}$ devido a mudanças extremas na geomorfologia e/ou vegetação regional. Estas taxas ocorrem tipicamente em lagos remotos ${ }^{49}$. Nas áreas estuarinas do litoral brasileiro, entretanto, taxas de sedimentação tão baixas nunca foram relatadas.

$\mathrm{Na}$ grande maioria das áreas estuarinas brasileiras em que foram determinadas taxas de sedimentação, estas variam, via de regra, de 0,11 a $0,98 \mathrm{~cm}$ ano ${ }^{-1}$ (Tabela 2). As maiores taxas são reportadas para complexos estuarino-lagunares, como no sistema lagunar do Estado do Rio de Janeiro ${ }^{54-56}$, onde variam de 0,28 a $0,76 \mathrm{~cm} \mathrm{ano}^{-1}$, e IguapeCananéia, no Estado de São Paulo ${ }^{53}$. Taxas intermediárias $(0,11-$ $0,49 \mathrm{~cm} \mathrm{ano}^{-1}$ ) foram reportadas para regiões estuarinas localizadas em áreas protegidas do litoral como nas Baías de Guanabara e Sepetiba $^{46,51}$ no Estado do Rio de Janeiro, e Todos os Santos, no Estado da Bahia $^{52}$. Taxas de sedimentação mais baixas são típicas de estuários oligotróficos ou que deságuam na plataforma continental ${ }^{53}$, sendo geralmente inferiores a $0,3 \mathrm{~cm}$ ano $^{-1}$.

Portanto, as camadas mais inferiores de colunas de sedimento de 0,5 a 1,0 m de profundidade integram geralmente a deposição de $\mathrm{Hg}$ ocorrida nos últimos 100 anos, ideais para a determinação dos valo- 
Tabela 2. Taxas de sedimentação de diferentes áreas estuarinas do litoral brasileiro

\begin{tabular}{|c|c|}
\hline Local & $\begin{array}{c}\text { Taxa de Sedimentação } \\
\left(\mathrm{cm} \mathrm{ano}^{-1}\right)\end{array}$ \\
\hline Enseada das Garças, Baía de Sepetiba (RJ) ${ }^{50}$ & $0,12-0,18$ \\
\hline Costa nordeste da Baía de Sepetiba $(\mathrm{RJ})^{51}$ & 0,5 \\
\hline Costa nordeste da Baía de Sepetiba (RJ) ${ }^{47}$ & $0,2-0,8$ \\
\hline $\begin{array}{l}\text { Estuário do Canal de São Francisco, } \\
\text { B. de Sepetiba }(\mathrm{RJ})^{48}\end{array}$ & 1,3 \\
\hline Baía de Todos os Santos $(\mathrm{BA})^{52}$ & 0,24 \\
\hline Parte interna da Baía de Guanabara (RJ ${ }^{46}$ & 0,49 \\
\hline Complexo estuarino-lagunar & $0,53-0,98$ \\
\hline Iguape-Cananéia $(\mathrm{SP})^{53}$ & \\
\hline Plataforma interna de Iguape-Cananéia $(\mathrm{SP})^{53}$ & $0,18-0,40$ \\
\hline Lagoa de Piratininga $(\mathrm{RJ})^{54}$ & $0,3-0,76$ \\
\hline Lagoa de Marica $(\mathrm{RJ})^{55}$ & $0,28-0,34$ \\
\hline Lagoa de Guarapina $(\mathrm{RJ})^{56}$ & $0,1-0,3$ \\
\hline
\end{tabular}

res de "background" da era pré-industrial brasileira, ocasião em que a urbanização não era intensa pois, somente a partir da década de 70 a população urbana superou a população rural no País. Tais testemunhos são de fácil coleta manual, transporte e preservação.

\section{“BACKGROUNDS” REGIONAIS DE Hg NA COSTA BRASILEIRA}

Resultados de $\mathrm{Hg}$ em perfis sedimentares com cerca de $1 \mathrm{~m}$ de profundidade não são tão abundantes quanto os em sedimentos superficiais, mas os resultados existentes confirmam os valores de "background" geoquímico de Hg na costa Leste e Sul da LME brasileira.

Na Tabela 3 são apresentados os resultados reportados na literatura, que mostram que os valores encontrados em profundidades características das deposições pré-industriais apontam para a costa Leste uma faixa de variação de 1 a $15 \mathrm{ng} \mathrm{g}^{-1}$ e para a costa Sul uma variação de 20 a $35 \mathrm{ng} \mathrm{g}^{-1}$, ambas na fração total dos sedimentos coletados.

Dessa forma, confirmam-se os valores mínimos encontrados em sedimentos superficiais como valores de "backgrounds" geoquímicos regionais e observa-se que na porção Leste o "background" encontrado para a fração $<63 \mu \mathrm{m}$ é muito similar ao encontrado para a fração total dos sedimentos coletados.

Como o número de resultados gerais ainda está abaixo do desejável para uma avaliação estatística, optou-se por considerar os valores máximos das faixas reportadas como os níveis de base característicos das respectivas regiões da costa. Ou seja, fica dessa forma esta- belecido que o "background" regional de $\mathrm{Hg}$ para a costa Leste é de $15 \mathrm{ng} \mathrm{g}^{-1}$, consideradas as determinações de $\mathrm{Hg}$ nas frações sedimentares $<63 \mu \mathrm{m}$ e/ou total, enquanto que para a costa Sul é de $35 \mathrm{ng} \mathrm{g}^{-1}$, consideradas as únicas determinações realizadas que utilizaram a fração total dos sedimentos costeiros.

\section{ÍNDICE DE GEOACUMULAÇÃO REGIONAL DE Hg NA COSTA BRASILEIRA}

Uma vez que fica confirmado pelos estudos realizados que o teor de $\mathrm{Hg}$ no folhelho médio não é o valor de base adequado para ser utilizado na avaliação da contaminação dos ambientes costeiros Leste e Sul brasileiros e que há na costa brasileira enriquecimento dos níveis de base de mercúrio, propõe-se a formulação do Índice de Geoacumulação Regional de $\mathrm{Hg}$ a partir dos "backgrounds" determinados nesse trabalho e através da seguinte fórmula:

Igeoregional $=\frac{\log \left(\mathrm{Cn} / \mathrm{B}_{\text {regional }}\right) / 0,301}{1,5}$

Sendo $\mathrm{Cn}$ a concentração medida de $\mathrm{Hg}$ na fração sedimentar de $<63$ $\mu \mathrm{m}$ ou total para a porção Leste da LME brasileira e na fração total

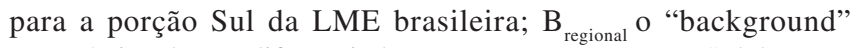
geoquímico de $\mathrm{Hg}$, diferenciado entre as porções Leste e Sul da LME brasileira e 1,5 o fator de correção para a possível variabilidade litológica dos sedimentos.

Esse fator sugerido preliminarmente propõe-se que seja mantido até que existam dados suficientes sobre a costa brasileira que permitam uma avaliação estatística da variabilidade dos valores naturais de $\mathrm{Hg}$ em sedimentos costeiros, não afetados por emissões antrópicas ou relativos a deposições pretéritas.

O fator de 0,301 é para converter o log de x para a base dois, como proposto originalmente ${ }^{14}$.

A partir dessa nova proposição são geradas novas faixas de variabilidades regionais da contaminação por $\mathrm{Hg}$, que determinam classes de Índices de Geoacumulação Regionais, dando-se ao grau mais elevado de contaminação valor 7 , que reflete para o caso da costa brasileira um enriquecimento de cerca de 200 vezes os valores de "backgrounds" regionais. Ou seja, enriquecimentos de cerca de 200 vezes os níveis de base são obtidos pela relação $1,5 \times 2^{7}$ (Tabela 4). Por outro lado, o grau mínimo (unitário) comprova a ausência de enriquecimento dos teores de $\mathrm{Hg}$ nos sedimentos costeiros (Tabela 5).

Na Figura 2 é apresentada a variabilidade da contaminação costeira de origem urbana e/ou industrial ao longo da costa brasileira, com caráter comparativo. Através desses resultados pode-se observar que as áreas da costa Leste e Sul da LME brasileira mais conta-

Tabela 3. Valores de "backgrounds" regionais determinados em perfis de testemunho ao longo da costa brasileira

\begin{tabular}{|c|c|c|c|}
\hline \multicolumn{4}{|c|}{ Litoral Leste da LME brasileira, segundo a tipologia de Ekau e Knoppers ${ }^{7}$} \\
\hline Local amostrado & $\begin{array}{l}\text { Máximo detectado } \\
\text { de } \mathrm{Hg}\left(\mathrm{ng} \mathrm{g}^{-1}\right)\end{array}$ & $\begin{array}{l}\text { Profundidade do } \\
\text { máximo de } \mathrm{Hg}(\mathrm{cm})\end{array}$ & $\begin{array}{l}\text { Valor de "background" } \\
\text { considerado }\left(\text { ng g g-1 }^{-1}\right.\end{array}$ \\
\hline Estuário R. Ceará (CE) este trabalho & 45 & 0 a 3 & 1 a 10 \\
\hline R. Jaguaribe (CE) este trabalho & 19 & 8 a 12 & $15 \pm 3$ \\
\hline \multicolumn{4}{|c|}{ Litoral Sul da LME brasileira, segundo a tipologia de Ekau e Knoppers ${ }^{7}$} \\
\hline Planície lamosa de manguezal, Baía de Sepetiba $(\mathrm{RJ})^{39}$ & 184 & 0 a 5 & 30 \\
\hline Canal de maré, Sepetiba $(\mathrm{RJ})^{39}$ & 98 & 0 a 5 & 30 \\
\hline Planície de lama, Baía de Sepetiba (RJ) ${ }^{41,57}$ & 137 & 0 a 5 & 20 a 30 \\
\hline Foz do Paraíba do Sul $(\mathrm{RJ})^{43}$ & 220 & 15 a 30 & 30 \\
\hline Baía de Guanabara $(\mathrm{RJ})^{42}$ & 890 & subsuperfície & $35 \pm 5$ \\
\hline
\end{tabular}


minadas por $\mathrm{Hg}$ oriundo de efluentes industriais e urbanos são a foz do R. Botafogo (PE) e o estuário do R. São João de Meriti, duas regiões que receberam por alguns anos efluentes de indústrias de cloro-soda. Nessas duas regiões os Índices de Geoacumulação culminaram nos graus mais elevados, 7 para a foz do R. Botafogo e 6 para o estuário do São João de Meriti, com fatores de enriquecimento da ordem de 207,0 e 96,6 vezes os teores de "backgrounds" regionais, denotando claramente a presença de uma potente fonte pontual de $\mathrm{Hg}$ nas regiões.

Em seguida, na ordem decrescente das regiões costeiras mais contaminadas por $\mathrm{Hg}$, encontramos a Baía de Guanabara, Baía de Todos os Santos e Lagoa dos Patos, contaminadas por fontes industriais e efluentes urbanos, bem como pelo chorume dos lixões. Isso

Tabela 4. Classificação dos Índices de Geoacumulação Regionais de $\mathrm{Hg}$, que determinam a contaminação costeira por efluentes urbanos e industriais (adotado a partir de Müller ${ }^{14}$ )

\begin{tabular}{cc}
\hline $\begin{array}{c}\text { Classes do } \\
\mathrm{I}_{\text {georegionais }}\end{array}$ & $\begin{array}{c}\text { Variabilidade do Índice de } \\
\text { Geoacumulação Regional }\end{array}$ \\
\hline 1 & $<0$ \\
2 & $0-1$ \\
3 & $1-2$ \\
4 & $2-3$ \\
5 & $3-4$ \\
6 & $4-5$ \\
7 & $>5$ \\
\hline
\end{tabular}

demonstra a importância e a dificuldade do controle de fontes difusas e corrobora a necessidade de um maior controle sanitário no País, tanto do ponto de vista do controle dos lixões, como do esgotamento doméstico e urbano.

Por outro lado, observa-se que áreas que servem como criatório de peixes e outros organismos marinhos estão contaminadas de forma

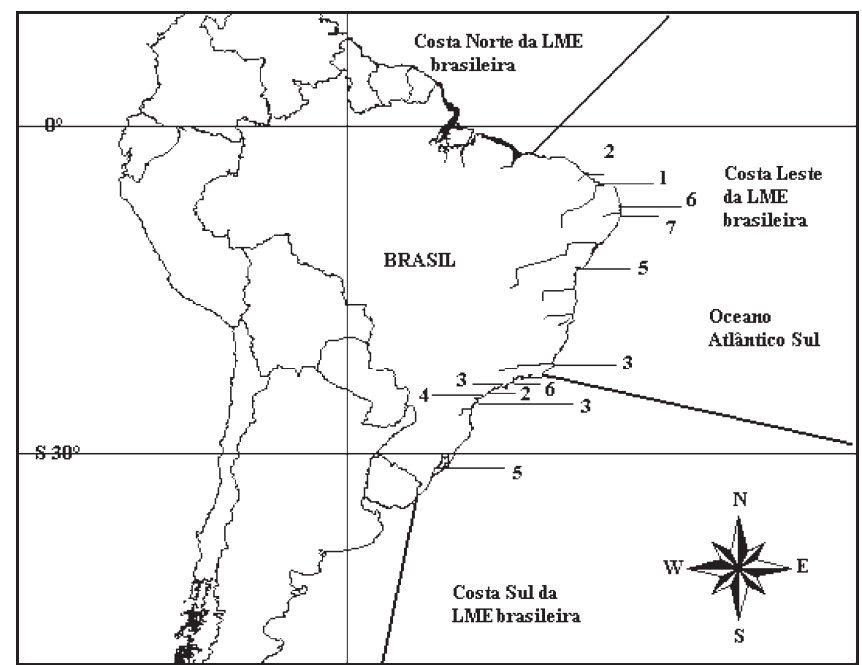

Figura 2. Tipologia da costa brasileira, adaptada de Ekau e Knoppers ${ }^{7}$, e sua contaminação por efluentes urbanos e industriais através do Índice de Geoacumulação Regional de $\mathrm{Hg}$, indicador ambiental

Tabela 5. Comparação da contaminação por atividades antrópicas da costa Leste e Sul da LME brasileira, utilizando-se Hg como indicador de qualidade ambiental e os teores máximos encontrados por local de coleta

Litoral Leste da LME brasileira, segundo a tipologia de Ekau e Knoppers ${ }^{7}$

\begin{tabular}{|c|c|c|c|}
\hline Local amostrado & $\begin{array}{l}\text { Fator máximo de } \\
\text { enriquecimento }\end{array}$ & $\begin{array}{c}\text { Índice de Geoacumulação } \\
\text { Regional }\left(\mathrm{I}_{\text {georegional }}\right)\end{array}$ & Classe do $\mathrm{I}_{\text {georegional }}$ \\
\hline Baía de Todos os Santos $(\mathrm{BA})^{28}$ & 55,0 & 3,8 & 5 \\
\hline Foz do Rio Botafogo $(\mathrm{PE})^{29}$ & 207,0 & 5,1 & 7 \\
\hline Foz do Rio Carrapicho (PE) ${ }^{29}$ & 87,0 & 4,3 & 6 \\
\hline Estuário R. Ceará-Maranguapinho $(\mathrm{CE})^{31-33}$ & 3,0 & 1,1 & 2 \\
\hline Estuário R. Cocó $(\mathrm{CE})^{33}$ & 3,0 & 1,1 & 2 \\
\hline Estuário R. Pacoti (CE) ${ }^{33}$ & 0,3 & $<0$ & 1 \\
\hline Estuário R. Jaguaribe $(\mathrm{CE})^{33}$ & 0,7 & $<0$ & 1 \\
\hline Estuário R. Ceará (CE) esse trabalho & 3,0 & 1,1 & 2 \\
\hline R. Jaguaribe $(\mathrm{CE})^{\text {esse trabalho }}$ & 1,3 & 0,2 & 2 \\
\hline
\end{tabular}

Litoral Sul da LME brasileira, segundo a tipologia de Ekau e Knoppers ${ }^{7}$

\begin{tabular}{|c|c|c|c|}
\hline Local amostrado & $\begin{array}{c}\text { Fator máximo de } \\
\text { enriquecimento }\end{array}$ & $\begin{array}{c}\text { Índice de Geoacumulação } \\
\text { Regional }\left(\mathrm{I}_{\text {georegional }}\right)\end{array}$ & Classe do $I_{\text {georegional }}$ \\
\hline Estuário de Rios, Baía de Sepetiba $(\mathrm{RJ})^{57}$ & 5,6 & 1,7 & 3 \\
\hline Baía de Sepetiba $(\mathrm{RJ})^{57}$ & 4,7 & 1,5 & 3 \\
\hline R. São João de Meriti (RJ) $)^{34}$ & 96,6 & 4,4 & 6 \\
\hline Planície de mangue R. Cubatão e Mogi (SP) ${ }^{35}$ & 1,7 & 0,5 & 2 \\
\hline Lagoa dos Patos $(\mathrm{RS})^{24}$ & 51,0 & 3,8 & 5 \\
\hline Estuário da Baía de Santos $(\mathrm{SP})^{10}$ & 16,4 & 2,7 & 4 \\
\hline Estuário da Baía de São Vicente $(\mathrm{SP})^{10}$ & 8,1 & 2,0 & 3 \\
\hline Baía de Santos $(\mathrm{SP})^{10}$ & 1,9 & 0,6 & 2 \\
\hline Planície lamosa, Baía de Sepetiba $(\mathrm{RJ})^{39}$ & 5,2 & 1,6 & 3 \\
\hline Canal de maré, Baía de Sepetiba $(\mathrm{RJ})^{39}$ & 2,8 & 1,0 & 2 \\
\hline Planície de lama, Baía de Sepetiba $(\mathrm{RJ})^{41,61}$ & 3,9 & 1,3 & 3 \\
\hline Foz do Paraíba do Sul $(\mathrm{RJ})^{43}$ & 6,3 & 1,8 & 3 \\
\hline Baía de Guanabara $(\mathrm{RJ})^{42}$ & 25,4 & 3,1 & 5 \\
\hline
\end{tabular}


significativa por mercúrio, um metal capaz de ser biomagnificado ao longo da cadeia trófica, o que pode explicar estimativas de contaminação do pescado em níveis acima dos recomendados pela $\mathrm{OMS}^{57-59}$.

Além disso, estudos realizados sobre a mobilização do $\mathrm{Hg}$ em ambiente costeiro demonstram que esse metal pode ser remobilizado de sedimentos mais profundos ${ }^{41,60,61}$ e alcançar a coluna d'água, onde se mantem associado, por exemplo, à matéria orgânica, passível de ser incorporado pela biota ${ }^{18,19,62,63}$.

\section{CONCLUSÃO}

O conteúdo de $\mathrm{Hg}$ em sedimentos pode ser utilizado adequadamente como um indicador da qualidade ambiental da costa brasileira e a variabilidade de graus do Índice de Geoacumulação Regional de $\mathrm{Hg}$ distingue a costa Leste da costa Sul da LME brasileira. Na região Sul, onde estão fortemente concentrados os grandes pólos industriais do País a maior parte das áreas estuarinas e costeiras estudadas estão contaminadas por efluentes urbanos e industriais, enquanto que na porção Leste ainda há áreas preservadas dessas contaminações.

Índices extremos do indicador denotaram áreas com presença de importante fonte pontual de $\mathrm{Hg}$.

$\mathrm{O}$ uso do $\mathrm{Hg}$ como indicador da qualidade ambiental da costa brasileira confirma que, uma vez controladas as contaminações de $\mathrm{Hg}$ oriundo das indústrias de cloro-soda que utilizavam $\mathrm{Hg}$ em células eletrolíticas, as fontes difusas, principalmente os lixões e o esgotamento doméstico e urbano, são as atividades antrópicas que mais necessitam de controle e adequação ao desenvolvimento sustentável da região costeira brasileira.

Os resultados obtidos e compilados da literatura mostram que a importância das fontes difusas vem crescendo nos últimos anos, junto com o aumento da população urbana adensada nas regiões costeiras do País.

\section{AGRADECIMENTOS}

À FUNCAP pelo suporte financeiro através das bolsas de mestrado de F. J. de Paula Filho e S. R. R. Maia. Ao CNPQ, pelas bolsas de IC e produtividade respectivamente de W. S. Marques, L. D. de Lacerda e R. V. Marins e pelo auxílio financeiro Proc. 466145/ 00-4. A tripulação do navio oceanográfico Prof. Martins Filho da UFC pelas realização e auxílio nas campanhas de coleta de sedimentos na Plataforma de Fortaleza, CE.

\section{REFERÊNCIAS}

1. Marins, R. V.; Freire, G. S. S.; Maia, L. P.; Lima, J. P. R.; Lacerda, L. D. Em South American Basins: LOICZ Global change assessment and synthesis of river catchment - Coastal Sea Interaction and Human Dimensions; Lacerda, L. D.; Kremer, H.; Salomons, W.; Kjerfve, B., eds.; LOICZ Reports \& Studies n²1: Texel, 2002, p. 92.

2. Lacerda, L. D.; Kjerfve, B.; Salomons, W.; Kremer, H. H. Em ref. 1, p. 7.

3. Rabelais, N. Em ref. 1, p. 77.

4. Esteves, F. A.; Fundamentos de Limnologia, $2^{\text {a }}$ ed., Interciência: Rio de Janeiro, 1999.

5. Ministério do Meio Ambiente, dos Recursos Hídricos e da Amazônia Legal; Perfil dos estados litorâneos do Brasil: Subsídios para a Implantação do Programa Nacional de Gerenciamento Costeiro, Brasília, Brasil, 1996.

6. Instituto Brasileiro de Geografia e Estatística; Indicadores de Desenvolvimento Sustentável - Brasil 2002, Dimensão Ambiental - Áreas Costeiras, Rio de Janeiro, Brasil, 2002.

7. Ekau, W.; Knoppers, B.; Arch. Fish. Mar. Res. 1999, 47, 109.

8. Medeiros, C.; Macêdo, S. J.; Feitosa, F. A. N.; Koening, M. L.; Arch. Fish. Mar. Res. 1999, 47, 133.

9. Valentini, E. Em Anais do II Encontro Nacional de Engenharia de Sedimentos, Rio de Janeiro, Brasil, 1996.
10. United Nations Environmental Programme; Overview on Land-based Sources and Activities Affecting the Marine, Coastal and Associated Freshwater Environment in the Upper Southwest Atlantic Ocean, UNEP Regional Seas Reports and Studies No. 170, The Hague, The Netherlands, 2000 .

11. International Oceanographic Commission; Second IOC Workshop on Sardine/Anchovy Recruitment Project (SARP) in the Southwest Atlantic, IOC Report no. 65, Paris, França, 1989.

12. Förstner, U.; Müller, G.; Schwermetalle in Flüssen und Seen als Ausdruck der Umweltverschmutzung, Springer Verlag: Heidelberg, 1974.

13. Förstner, U.; Wittman, G. T. M.; Metal Pollution in the Aquatic Environment, Springer Verlag: Heidelberg, 1981.

14. Salomons, W.; Förstner, U.; Metals in the Hydrocycle, Springer Verlag: Heidelberg, 1984.

15. Occupational Safety and Health Administration.; OSHA Instruction CPL 2-26, 6 CH-1, Washington, 1978.

16. Clarkson, T. W. Em Mercury Pollution: Integration and Synthesis; Watras, C. I.; Huckabee, J. W., eds.; Lewis Publishers: Boca Raton, 1994, p. 631.

17. Marins, R. V.; Lacerda, L.; Villas Boas, R. C. Em Mercury Contamination Sites: Characterization, Risk Assessment and Remediation, Ebinghaus, R.; Turner, R. R.; Lacerda, L. D.; Vasiliev, O.; Salomons, W., eds.; Springer Verlag: Berlin, 1999.

18. Lacerda, L. D.; Silva, L. F. F.; Marins, R. V.; Mounier, S.; Paraquetti, H. H. M.; Benaim, J.; Wetlands Ecol. Management 2001, 9, 323.

19. Lacerda, L. D.; Paraquetti, H. H. M.; Marins, R. V.; Mounier, S.; Benaim, J.; Frevier, D.; J. Braz. Chem. Soc. 2001, 12, 93.

20. Molisani, M. M.; Marins, R. V.; Machado, W.; Paraquetti, H. H. M.; Bidone, E.; Lacerda, L. D.; Reg. Environ. Change 2004, 4, 17.

21. Sobreira, F. G.; Fonseca, M. A.; Pires, F. R. M.; An. Acad. Bras. Ci. 1987, $59,363$.

22. Lacerda, L. D.; Marins, R. V.; J. Geochem. Explor. 1997, 58, 223.

23. Environmental Protection Agency; Locating and Estimating Air Emissions from Sources of Mercury and Mercury Coumpounds. Rep. EPA/454/R-93, Research Triangle Park, North Carolina, 1993.

24. Mirlean, N.; Andrus, V. E.; Baisch, P.; Mar. Pollut. Bull. 2003, 46, 331.

25. Thornton, J. D.; Eisenreich, S. J.; Munger, J. W.; Gorham, E. Em Atmospheric Pollutants in Natural Waters; Ann Arbor Sci. Publ. Inc.: Michigan, 1981.

26. Marins, R. V.; Silva Filho, E. V.; Lacerda, L. D.; J. Braz. Chem. Soc. 1996, 7, 177.

27. Lacerda, L. D.; Paraquetti, H. H. M.; Rezende, C. E.; Silva, L. F. F.; Silva Filho, E. V.; Marins, R. V.; Ribeiro, M. G.; J. Braz. Chem. Soc. 2002, 13,165 .

28. Pfeiffer, W. C.; Fiszman, M.; Lacerda, L. D. Em Metals in the Coastal Environments of Latin America; Seeliger, U.; Lacerda, L. D.; Patchineelan, S. R., eds.; Springer Verlag: Berlin, 1988, p. 16.

29. Meyer, U.; Tese de Doutorado, Zentrum für Marine Tropenökologie, Alemanha, 1996.

30. Müller, G.; Irion, G.; Morais, J. O.; Tintelnot, M.; CD room $3^{\text {rd }}$ Int. Symp. Environmental Geochemistry in Tropical Countries, Nova Friburgo, Brasil, 1999.

31. Marins, R. V.; Lacerda, L. D.; Mounier, S.; Paraquetti, H. H. M.; Marques, W. S.; Geochim. Brasiliensis 2002, 16, 27.

32. Marques, W. S.; Monografia de Graduação, Universidade Federal do Ceará, Brasil, 2002

33. Vaisman, A. G.; Tese de Mestrado, Universidade Federal do Ceará, Brasil, 2003.

34. Barrocas, P. R. G.; Wasserman, J. C. Em Environmental Geochemistry in the Tropics; Wasserman, J. C.; Silva-Filho, E. V.; Villas-Boas, R. C., eds.; Springer: Berlim, 1998, p. 57.

35. Ferrer, L. M.; Hypolito, R.; CD Room VII Congresso Brasileiro de Geoquímica da Sociedade Brasileira de Geoquímica, Curitiba, Brasil, 2001.

36. Förstner, U.; Contaminated Sediments, Springer Verlag: Berlim, 1989.

37. Salomons, W.; Stigliani, W. M.; Biogeodynamics of Pollutants in Soils and Sediments, Springer: Berlim, 1995.

38. Eysink, G. G. J.; Ambiente CETESB 1988, $2,6$.

39. Silva, L. F. F.; Machado, W.; Lisboa, S. D.; Lacerda, L. D.; Water Air Soil Pollut. 2003, 145, 67.

40. Luiz-Silva, W.; Matos, R. H. R.; Kristosch, G. C.; Quim. Nova 2002, 25, 753.

41. Marins, R. V.; Lacerda, L. D.; Gonçalves, G. O.; de Paiva, E. C.; Bull. Environ. Contam. Toxicol. 1997, 58, 733.

42. Machado, W.; Moscatelli, M.; Rezende, L. G.; Lacerda, L. D.; Environ. Pollut. 2002, 120, 455 .

43. Lacerda, L. D.; Carvalho, C. E. V.; Rezende, C. E.; Pfeiffer, W. C.; Mar. Pollut. Bull. 1993, 26, 220. 
44. Matschullat, J.; Ottgenstein, R.; Reiman, C.; Environ. Pollut. 2000, 39, 990.

45. Birch, G. F.; Robertson, E.; Taylor, S. E.; McConchie, D. M.; Environ. Pollut. 2000, 39, 1015

46. Godoy, J. M.; Moreira, I.; Bragança, M. J.; Wanderley, C.; Mendes, L. B.; J. Radioanal. Nucl. Chem. 1998, 227, 157.

47. Forte, C. M. S.; Tese de Mestrado, Universidade Federal Fluminense, Brasil, 1996.

48. Barcellos, C.; Tese de Doutorado, Universidade Federal Fluminense, Brasil, 1995.

49. Lacerda, L. D.; Ribeiro, M. G.; Cordeiro, R. C.; Siffedine, A.; Turcq, B.; J. Braz. Soc. Adv. Sci. 1999, 51, 363.

50. Smoak, S.; Pachineelam, S. R.; Mangroves and Salt Marshes 1999, 3, 98.

51. Leitão Filho, C. M.; Silva Filho, E. V.; Wasserman, J. C.; Patchineelam, S. R.; CD-ROM Anais do $5^{\circ}$ Congresso Brasileiro de Geoquímica, Niterói, Brasil, 1995.

52. Lessa, G. C.; Bettencourt, A. C. S. P.; Brichta, A.; Dominguez, J. M. L.; An. Acad. Bras. Ci. 2001, 72, 573.

53. Saito, R. T.; Figueira, R. C. L.; Tessler, M. G.; Cunha, I. I. L.; J. Radioanal. Nucl. Chem. 2001, 250, 109.

54. Huang, W.; Campredon, R.; Bernat, M.; Latouche, C.; Environ. Geol. 1994, $23,241$.
55. Fernex, F. E.; Bernat, M.; Ballestra, S.; Fernandes, L.; Marques, A. N.; Hydrobiologia 1992, 242, 69.

56. Machado, E. C.; Knopper, B. A.; Sci. Total Environ. 1988, 75, 341.

57. Marins, R. V.; Lacerda, L. D.; Paraquetti, H. H. M.; Paiva, E.C.; Villas Boas, R. C.; Bull. Environ. Contam. Toxicol. 1998, 61, 57.

58. Lacerda, L. D.; Paraquetti, H. H. M.; Marins, R. V.; Rezende, C. E.; Zalmon, I. R.; Gomes, M. P.; Farias, V.; Rev. Brasil. Biol. 2000, 60, 1.

59. Kehrig, H. A.; Costa, M.; Moreira, I.; Malm, O.; Mar. Pollut. Bull. 2002, 44, 1018.

60. Marins, R. V.; Lacerda, L. D.; Mounier, S.; CD Rom Proc. Silver Anniversary Int. Conf. on Heavy Metals in the Environment, Ann Arbor, USA, 2000.

61. Marins, R. V.; Lacerda, L. D.; CD Rom VII Congresso Brasileiro de Geoquímica da Sociedade Brasileria de Geoquímica, Porto Seguro, Brasil, 1999.

62. Mounier, S.; Lacerda, L. D.; Marins, R. V.; Benaim, J.; Bull. Environ. Contam. Toxicol. 2001, 67, 519.

63. Marins, R. V.; Lacerda, L. D.; Paraquetti, H. H. M.; CD Rom Proc. Silver Anniversary Int. Conf. on Heavy Metals in the Environment, Ann Arbor, USA, 2000. 